\title{
Prognostic characterization of the pyroptosis- related subtypes and tumor microenvironment infiltration in glioma
}

Jiarong He

Second Xiangya Hospital

Jiaoying Jia

Second Xiangya Hospital

Jiawei Lei

Second Xiangya Hospital

Jingyu Yu

Huashan Hospital Fudan University

Wen Zhou

Central South University

Mingming Zhang ( $\nabla$ zhangmm@csu.edu.cn )

Central South University https://orcid.org/0000-0002-1261-1672

\section{Research Article}

Keywords: glioma, pyroptosis, mutation burden, signature, tumor microenvironment

Posted Date: December 29th, 2021

DOI: https://doi.org/10.21203/rs.3.rs-1212592/v1

License: () (1) This work is licensed under a Creative Commons Attribution 4.0 International License.

Read Full License 


\title{
Prognostic characterization of the pyroptosis-related subtypes and tumor microenvironment infiltration in glioma
}

\author{
Jiarong $\mathrm{He}^{1}$, Jiaoying $\mathrm{Jia}^{1}$, Jiawei Lei ${ }^{2}$, Jingyu $\mathrm{Yu}^{3}$, Wen Zhou ${ }^{1}$, and Mingming \\ Zhang ${ }^{1 *}$
}

1 Department of Neurosurgery, The Second Xiangya Hospital, Central South University, Changsha, Hunan 410011, PR China.

2 Department of General Surgery, The Second Xiangya Hospital, Central South University, Changsha, Hunan 410011, PR China.

3 Department of Neurosurgery, Huashan Hospital, Shanghai Medical College, Fudan University, Shanghai 200040, PR China.

\section{*Correspondence:}

Mingming Zhang: zhangmm@csu.edu.cn

\section{ABSTRACT}

Background: Pyroptosis could regulate tumor cell trafficking, invasion, and metastasis, as well as tumor microenvironment (TME). However, prognostic characteristics of pyroptosis-related genes (PRGs) and their impact on the progression of glioma remain insufficient.

Methods: The genetic, transcriptional, and survival data of glioma patients used for bioinformatics analysis were obtained from the Chinese Glioma Genome Atlas (CGGA) and The Cancer Genome Atlas (TCGA) databases.

Results: We first screened two different molecular subtypes and found that PRG variations were associated with the characteristics of TME cell infiltration, clinicopathological characteristics, and prognosis of patients with glioma. After Cox regression of differentially expressed genes, a risk-score for predicting overall survival (OS) and progression-free survival (PFS) was calculated and its predictive accuracy in glioma patients was then validated. The high-risk group of PRGs signature showed a significantly poor OS compared to the low-risk group (training cohort, $p<0.001$, validation cohort, $p<0.001$ ). A high risk-score implies more immune cell infiltration and a better immunotherapy response to immune checkpoint blockers. Furthermore, the risk-score was significantly associated with the chemotherapeutic drug sensitivity and cancer stem cell (CSC) index. Subsequently, we established a highly accurate nomogram to facilitate the applicability in the preliminary clinical application of the risk-score. 
Conclusion: Our findings may lay the foundation for future research targeting pyroptosis in glioma and pave the way for evaluating prognosis and developing more effective immunotherapy strategies.

Keywords: glioma; pyroptosis; mutation burden; signature; tumor microenvironment;

\section{INTRODUCTION}

Glioma is the leading malignant tumor, accounting for about $30 \%$ of tumors and $81 \%$ of notorious malignancy in the central nervous system (1). According to the molecular markers and histology characteristic of glioma, the World Health Organization classification divides glioma into low-grade glioma (LGG, grade II-III) and glioblastoma multiforme (GBM, grade IV), most of LGG progresses to GBM, with a five-year survival rate of only $5 \%(2,3)$. In recent decades, survival rates have improved significantly with the upgrading of equipment, multidisciplinary therapeutic regimes as well as the improvement of early screening systems (4). However, the prognosis of glioma remains unsatisfactory, the potential therapeutic targets and independent prognostic biomarkers must be identified.

Pyroptosis, referred to as programmed cell death, is a novel form of cellular inflammatory necrosis (5). Triggered by certain inflammasomes, Pyroptotic cells rely on the activation of inactive cytokines and cleavage of the gasdermin (GSDM) protein superfamily (GSDM A-E and DFNB59) (6). In terms of mechanism, canonical, non-canonical, caspase-3/8-mediated, and granzyme-mediated inflammasome were all involved in pyroptosis (7). Pyroptosis was originally characterized and identified to be a crucial role against infection, and emerging evidence suggests that it is also involved in the process of tumors. It has been described that GSDM proteins, proinflammatory cytokines, and vesicles, which are the main bioactive components of pyroptosis, are related to tumorigenesis, metastatic progression, and inflammatory tumor microenvironment (8). More recently, further headway has been made into pyroptosis. Hou et al. reported that accumulated p-STAT3 stimulated PD-L1 nuclear translocation under hypoxia conditions, thereby enhancing the expression of GSDMC. The activation of caspase- 8 by macrophage-derived TNF- $\alpha$ can efficiently cleave GSDMC into N-GSDMC, which eventually leads to pyroptosis (9). In addition, unlike apoptosis, multiple risk-related signaling cytokines and molecules are activated and released when pyroptosis occurs, accompanied by activation of the immune-inflammatory response system (IRS) (10). Zhou et al. also showed the decisive role of $\mathrm{NK}$ cells in the induction of antitumor immune responses, predominantly mediated by pyroptosis (11). The TME is widely involved in the occurrence and development of malignant tumors by targeting pyroptosis (12). Apart from invading tumor cells, the TME also contains lymphatic endothelial cells, fibroblasts, extracellular matrix (ECM), immune and inflammatory cells, diffusible chemokines and cytokines secreted from neighboring tumor and stromal cells. An increasing number of researches also have shown that complex crosstalk between the TME and pyroptosis $(13,14)$. Currently, most studies only evaluate one or two PRGs due to technical limitations, while the antitumor activity is characterized by highly 
coordinated interactions of multiple genes. Therefore, a comprehensive analysis of the features of multiple PRG-mediated cells infiltrates may provide significant insights into the underlying mechanisms of glioma tumorigenesis and the prediction of immunotherapy responses.

Given the current findings, we know that pyroptosis is involved in tumor development and the anti-tumor process, but there are few studies on its specific prognostic characteristics in glioma. Thus, we comprehensively explored the genetic and transcriptional profiles of PRGs and presented a systematic overview of the glioma immune microenvironment, coupled with computational analysis. First, we divided 667 glioma patients into two distinct subtypes based on the expression level of PRG. All the samples in training (TCGA) and validation (CGGA) cohorts were classified into two discrete groups based on the 3 survival-related PRGs identified by Lasso-penalized Cox regression. We then identified the immune landscape of glioma and developed a scoring model for predicting overall survival, which accurately predicted patient prognosis and guide optimal treatment choices for glioma.

\section{MATERIALS AND METHODS}

\section{Datasets}

The workflow diagram of this research was shown in Figure S1. We obtained the transcriptional landscape of 1431 normal brain samples from the Genotype-Tissue Expression (GTEx) Data Portal (https://xenabrowser.net/datapages/) (15). The RNA-sequencing (RNA-seq) data (fragments per kilobase million, FPKM) and corresponding clinicopathological features of 667 glioma patients were obtained from the TCGA database (https://portal.gdc.cancer.gov/repository). The CGGA: mRNAseq693 dataset from the CGGA database (http://www.cgga.org.cn/) was used as the external validation cohort (16). Patients without survival information were excluded from further analysis. The detailed information of these samples is presented in Table S1. The FPKM values of glioma were converted into transcripts per kilobase million (TPM) before further investigation (17). All the datasets were available from the previously published literature and the ethics statement confirmed that all written informed consent was obtained.

\section{Identification of differentially expressed PRGs and Consensus clustering}

In this study, a total of 40 PRGs were downloaded from the human gene database (GeneCards) (https://www.genecards.org/) and previous publications, which are provided in Table S2 (18-20). The mRNA expression data of the two cohorts were normalized using the "edger" $\mathrm{R}$ package before comparison. To explore the main DEGs between glioma tissues and normal brain tissues, the "limma" $\mathrm{R}$ package was utilized with $|\log 2 \mathrm{FC}| \geq 1$ and FDR $<0.05$ in the TCGA cohort. $\mathrm{P}$ values were set as follows: * $\mathrm{P}<0.05$, ** $\mathrm{P}<0.01$, and $* * * \mathrm{P}<0.001$. PPI network of DEGs was constructed by using interactive gene retrieval tool, STRING, version 11.5 (https://string-db.org/). Consensus clustering was employed to examine two distinct pyroptosis-mediated patterns by the k-means method. To ensure the stability of our 
classification, we use the "ConsensuClusterPlus" package to control the number and stability of clusters. In addition, the differences in OS and PFS among the two subtypes were evaluated by Kaplan-Meier method using the "survminer" package in R.

\section{Correlations of two subtypes with TME and PD-1/PD-L1 blockade in glioma}

We assessed the stromal/immune cells of each glioma sample using the ESTIMATE algorithm. Furthermore, the CIBERSORT algorithm was used to calculate the fraction of 22 immune cell subsets of each glioma patient (21). The infiltration levels of immune cell subsets in glioma TME were explored by a single-sample GSEA algorithm (22). Also, the expression of PD-1/PD-L1 blockade between the two subtypes was analyzed.

\section{Development and validation of a pyroptosis-related prognostic model}

Univariate and multivariate Cox proportional hazard regressions were used to evaluate the prognostic factors, including age, gender, grade, and risk-score. For Kaplan-Meier curves, the survival rates among two groups were compared by the log-rank test. Hazard ratios (HR) and 95\% confidence intervals (CI) were calculated using the "forestplot" $\mathrm{R}$ package. Based on prognostic DEGs, the Lasso-penalized Cox regression algorithm (R package "glmnet") was used to construct a prognostic model. We set the cut-off value as 0.05 , and 3 PRGs were selected for further analysis.

The risk-score was calculated using the following equation:

risk-score $=\Sigma(\operatorname{Exp} *$ Coef $)$

where Exp and Coef represent the expression and risk coefficient of each PRG, respectively. The risk score of the gene was calculated after centralized standardization of the mRNA expression data (using the "scale" function in R). By applying the median filter on the risk score, patients with glioma were divided into high/low-risk subgroups. and the OS of the two subgroups was compared by the Kaplan-Meier method. Principal component analysis (PCA) was performed based on 3-gene signatures, using the "prcomp" function in R. Predictive capability of the risk scores was assessed by time-dependent Receiver Operating Characteristic (ROC) curve analysis. The "timeROC" and "survminer" R packages were used to perform the 1-, 3-, and 5-year sensitivity curve analyses.

\section{Independent prognostic values of pyroptosis-related genes}

The phenotypic information (age, gender, and grade) of glioma patients was extracted from the TCGA and CGGA cohorts. These clinical variables were analyzed in conjunction with the risk score in a Cox proportional hazards regression model. Based on the median risk score of the TCGA cohort, glioma patients in the CGGAmRNAseq693 cohort were also classified into two groups, and then these subgroups were compared to validate the prognostic model.

\section{Immunohistochemistry (IHC) Staining}


To assess differences in DEGs expression at the protein level, Immunohistochemistry staining images of CASP4, CASP6, and GZMB protein expression in glioma tissues and normal brain tissues were analyzed from the HPA database (http://www.proteinatlas.org/).

\section{Immune infiltration and Functional enrichment analysis for DEGs}

Based on these DEGs, R packages " clusterProfiler " was used for Gene Ontology (GO) and Kyoto Encyclopedia of Genes and Genomes (KEGG) analysis. In addition, ssGSEA was performed using the "GSVA" R package to calculate the scores of tumor-infiltrating immune cells and immune-related pathways.

\section{Mutation, Drug susceptibility, and Cancer stem cell (CSC) index analysis}

To identify somatic mutations in glioma patients between the two groups, the "maftools" R package was used to generate mutation annotation format (MAF) profiles (derived from VarScan2). The Tumor mutation burden (TMB) scores for each sample in the high- and low-risk groups were also calculated. To investigate the difference in the therapeutic efficacy of chemotherapy drugs between the two risk groups, we used the "pRRophetic" $\mathrm{R}$ package to calculate the half-inhibitory concentration (IC50) value of chemotherapy drugs newly used to treat glioma. Furthermore, we examined the relationship between the CSC index and two groups.

\section{Establish the nomogram scoring model}

Based on the results of the independent prognostic analysis, the risk score and clinical parameters were used to construct a predictive nomogram model using the "rms" package. Each clinicopathologic variable was computed with a score in the nomogram scoring model, and the scores of all variables in each sample were added up to get the final score. Clinical ROC and Decision curve analysis (DCA) were performed to assess the effectiveness of the nomogram model. Calibration curves of the model were used to describe the predicted power for the probability of survival events at 3and 5-years.

\section{Statistical Analysis}

Wilcoxon matched-pairs signed rank test was utilized to compare the transcriptional variations between the normal brain and glioma tissues, Mann-Whitney test was used to compare the infiltration of immune cells and activation of immune pathways between the two subgroups. The paired log-rank test was applied to compare the overall survival between subgroups. Pearson correlation test was used in statistics to evaluate the correlation coefficient. $\mathrm{P}<0.05$ was considered statistically significant. Other statistical methods are described above. All statistical analyses were accomplished with R software (v4.0.2). 


\section{RESULTS}

\section{Identification of genetic alterations and transcriptional}

\section{variations of PRGs in glioma}

Gene expression levels of 40 PRGs were measured between 667 tumor and 1431 normal tissues from TCGA-GBM/LGG and GTEx-Brain data. A total of 33 PRGs were either downregulated or upregulated in glioma (all FDR $<0.001$, Figure 1A). Among them, 15 genes (CASP4, GPX4, GSDMB, GSDMD, GZMB, HMGB1, IL6, IRF1, NLRP1, NLRP2, NOD1, PJVK, PLCG1, PRKACA, and SCAF11) were downregulated while 18 other genes (AIM2, BAK1, BAX, CASP1, CASP3, CASP6, CASP8, GSDME, GZMA, IL18, IL1B, IRF2, NLRC4, NLRP3, NOD2, PYCARD, TIRAP, and TNF) were enriched in glioma compared with normal brain tissues (Table S3).

In addition, we identified the location of alterations of the PRGs featuring copy number variation (CNV) on chromosomes (Figure 1B). The CNV alteration frequency was also investigated, which revealed more than half of the 40 PRGs were widespread with a copy number deletion (Figure 1C, E). Then we explored the incidence of somatic mutations and copy number variations of 40 PRGs in GBM and LGG. At the genetic level, 43 of 390 (11.03\%) GBM samples and 30 of 506 (5.93\%) LGG samples confirmed genetic mutations. Figure 1D demonstrated that NLRP3 with the highest frequency of variants in GBM, followed by NLRP7, NLRP2, and NOD1. Compared to the LGG cohort, NLRP2 showed the highest mutation frequency, among the 40 PRGs (Figure 1F). The correlation network containing all PRGs and DEGs were presented in (Figure S2A) and (Figure S2C), respectively. We further found some PRGs with downregulated mRNA expression, such as NLRP2 and HMGB1, showed CNV loss, indicating that $\mathrm{CNV}$ alteration might regulate the transcriptional activity of PRGs.

To further explore the potential interactions among these pyroptosis-related genes, a protein-protein interaction (PPI) analysis was constructed to detect these PRGs interactions with a minimum interaction score of 0.9 (the highest confidence), which revealed that NLRP1, CASP5, GSDMD, NLRC4, PYCARD, CASP1, and NLRP3 were hub genes (Figure S2B). Our analysis observed significant differences in both the landscape of genetic alterations and transcriptional variations of PRGs between control and glioma samples, suggesting the potential function of PRGs in glioma oncogenesis. 


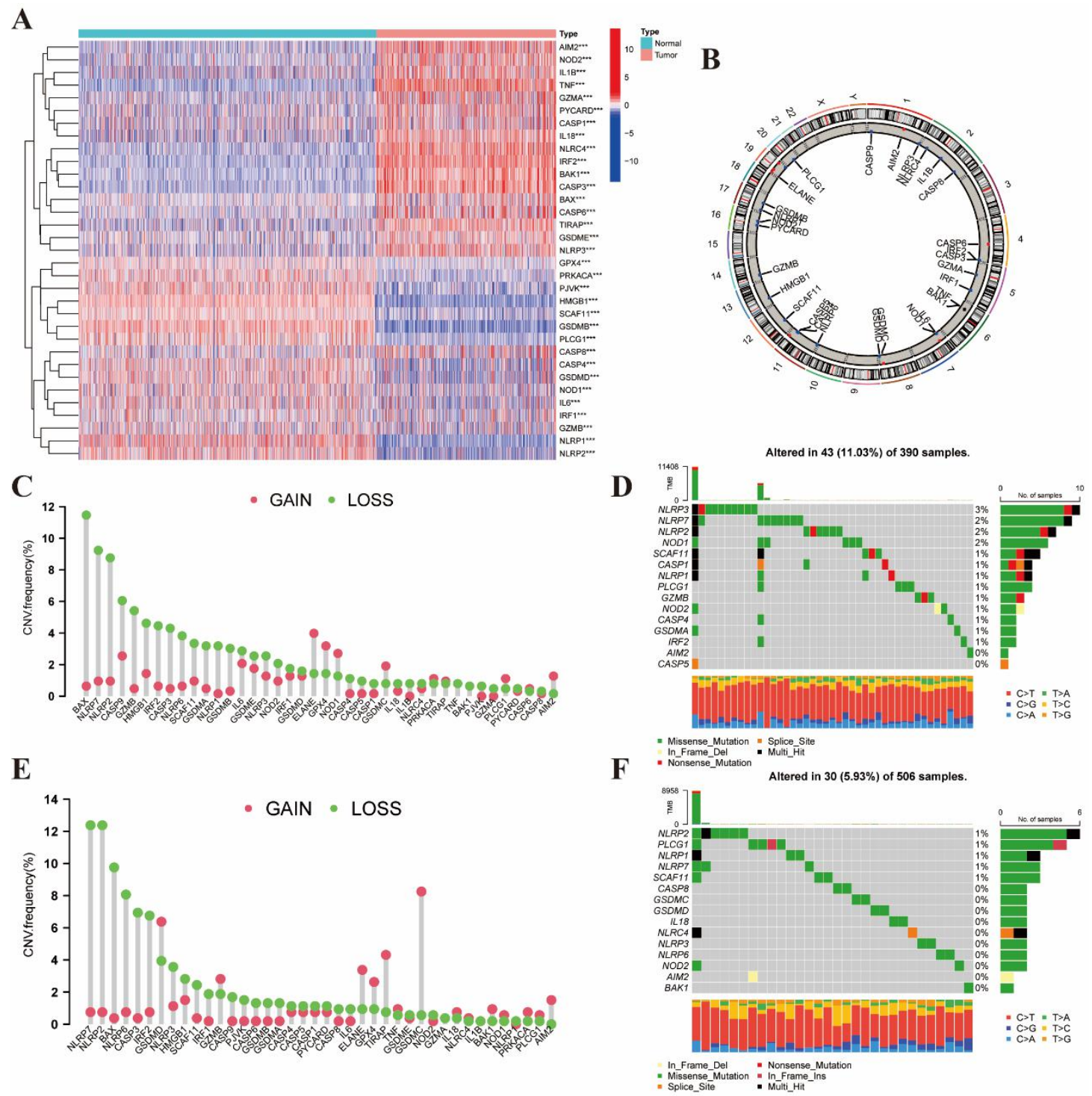

FIGURE 1 Characteristics and differences of PRGs in glioma. (A) Heatmap (red: high expression; blue: low expression) of 33 DEGs in glioma tissues and normal brain tissues (red, Tumor; blue, Normal). $\mathrm{P}$ values were set as: $* \mathrm{P}<0.05 ; * * \mathrm{P}<0.01$; ***P $<0.001$. (B) The alterations of CNV locations in PRG on 23 chromosomes. The CNV variation frequency of PRGs in GBM cohort (C) and LGG cohort (E). The height of the column indicated the alteration frequency. The landscape of somatic mutation frequency of PRGs in GBM cohort (D) and LGG cohort (F). Each waterfall plot showed the mutation information of the top 15 PRGs.

\section{Identification of pyroptosis subtypes in glioma and its TME}

\section{characteristics}

The comprehensive landscape of 33 DEG interactions, connections, and their prognostic significance in patients with glioma was presented in a pyroptosis network (Figure 2A and Table S4). To explore the relationship between the expression of the 
33 differentially expressed pyroptosis genes and glioma subtypes, we performed the consensus cluster analysis to classify glioma patients. By applying the K-means clustering algorithm, the intra-group correlation was the highest and the inter-group correlation was low when $\mathrm{k}=2$. The results indicated that the 667 glioma patients were divided into two clusters (cluster $\mathrm{C} 1, \mathrm{n}=472$; cluster $\mathrm{C} 2, \mathrm{n}=195$ ) based on the 33 DEGs (Figure 2B).

We also assessed the TME score (StromalScore, ImmuneScore, and ESTIMATEScore) of the two clusters using the ESTIMATE package. A higher StromalScore or ImmuneScore indicated a higher relative content of stromal or immune components in the immune microenvironment, while ESTIMATEScore represented the accumulation of stromal or immune cells. We observed that patients with subtype C2 had a higher TME score (Figure 2C). Correspondingly, analysis of the immune checkpoints indicated that PD-1 and PD-L1 were highly expressed in subtype C2 (Figure 2D, E). Next, a significant difference was found in PFS and OS between the two clusters (Figure 2F, G, $P<0.001$,). The gene expression profile (GEP) and corresponding clinicopathological parameters including the degree of tumor histological differentiation (G2-G4), age ( $\leq 65$ or $>65$ years), and gender (male or female) were presented in a heatmap. Figure $\mathbf{2 H}$ showed that cluster $\mathrm{C} 1$ was preferentially related to lower Age $(p<0.05)$ and lower Grade $(p<0.05)$ compared to those in cluster $\mathrm{C} 2$.

To investigate the effect of PRGs on the tumor immune microenvironment of glioma, we evaluated the tumor-infiltrating immune levels of every glioma sample between the two subtypes using the CIBERSORT algorithm (Table S5). Our study indicated that cluster $\mathrm{C} 1$ showed higher infiltration fractions of T cells CD4 memory resting, NK cells activated, Monocytes, and Mast cells activated, whereas cluster C2 was more associated with $\mathrm{T}$ cells $\mathrm{CD} 8$, Mast cells resting, Macrophages M1, and Macrophages M2 (Figure 2I). 
A

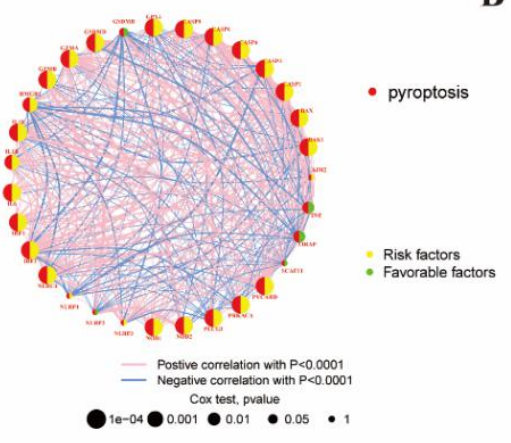

D

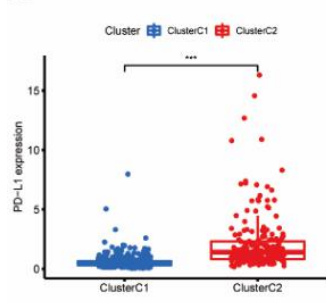

E

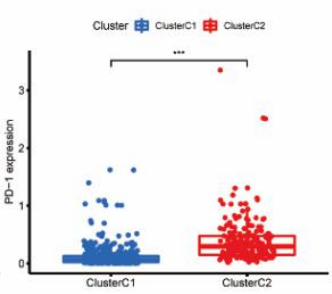

F
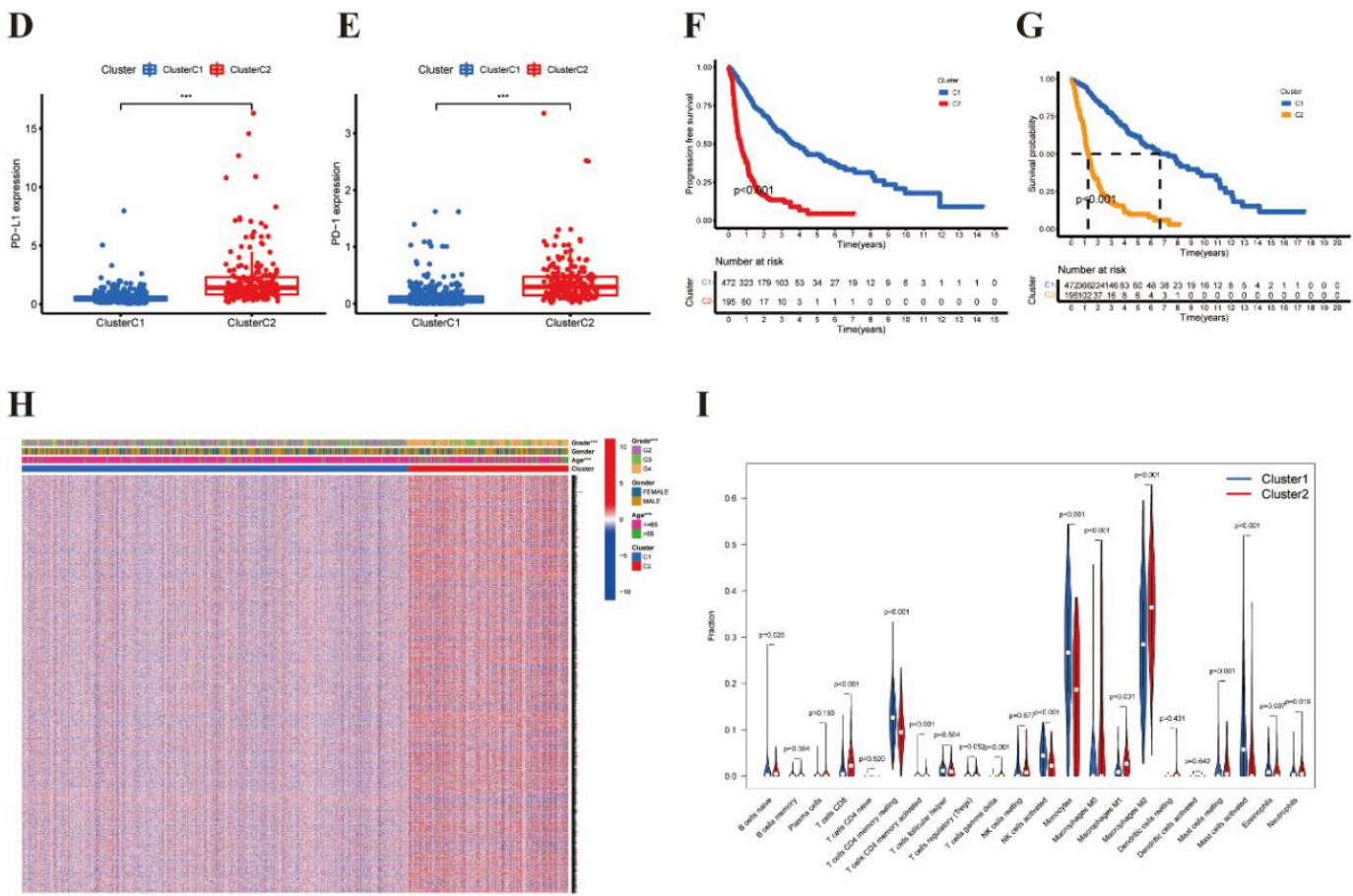

G

I

C
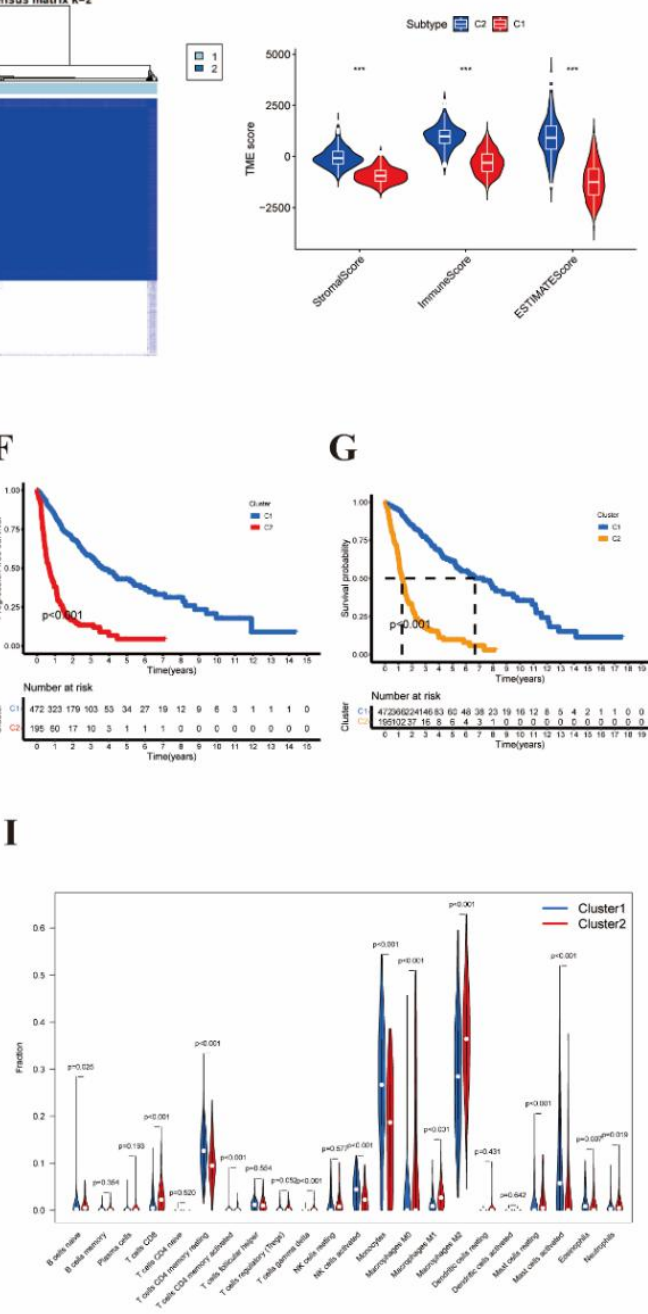

FIGURE 2 Clinicopathological and TME characteristics of PRG subtypes based on DEGs. (A) Interactions among 33 DEGs in glioma. The thickness of the lines represents the strength of the correlation between the PRGS. Pink and blue represent positive and negative correlations, respectively. (B) The consensus clustering matrix of 667 samples in the TCGA cohort $(\mathrm{k}=2)$. (C) Associations between the two clusters and TME score. Kaplan-Meier survival curves for PFS (D) and OS (E) of the two clusters (log-rank tests, $p<0.001)$. (F, G) PD-1/PD-L1 expression in the two glioma clusters. (H) Heatmap of the clinicopathologic features classified by these DEGs. (I) The infiltrating scores of 22 immune cells in the two clusters.

\section{Construction and utilization of the pyroptosis-related prognostic model}

To construct a PRGs-Based prognostic model, we used univariate and multivariable Cox regression analysis to screen for survival-related genes. Univariate Cox regression showed the prognostic value of 26 PRGS for glioma patients $(p<0.05$, 
Figure 3A and Table S6). Then, a total of 10 genes (CASP3, CASP4, CASP4, GZMB, IL18, PLCG1, PRKACA, CASP1, TNF, and GZMA) with independent prognostic value identified by multivariate Cox regression, and among them, 7 genes (CASP3, CASP4, CASP4, GZMB, IL18, PLCG1, and PRKACA) were associated with an increased likelihood of death (HR $>1$ ), while the remaining 3 gene (CASP1, TNF, and GZMA) was a protective factor with HR $<1$ (Figure 3B and Table S6).

Lasso-penalized Cox regression analysis was applied to construct a three pyroptosis-based prognostic signature based on the optimum $\lambda$ value (Figure 3C, D). The risk score of the pyroptosis-based model was calculated as follows: Risk score $=$ $(0.5826 *$ CASP4 exp. $)+(0.6889 *$ CASP6 exp. $)+(0.1098 *$ GZMB exp. $)$. Patients were divided into high-risk group $(\mathrm{n}=333)$ and low-risk group $(\mathrm{n}=334)$ according to the median cut-off value (Figure 3E). Kaplan-Meier survival analysis revealed that the survival probability of glioma patients in the low-risk group was significantly higher than that in the high-risk group (Figure 3F, $P<0.001$ ). Furthermore, time-dependent ROC analysis was performed to evaluate the effectiveness of the actuarial risk assessment model, and the area under the curve (AUC) for the three pyroptosis-related genes model was 0.855 for 1 -year, 0.881 for 3-year, and 0.826 for 5-year survival (Figure 3G). The principal component analysis and t-distributed Stochastic Neighbor Embedding (t-SNE) revealed that patients with different risk scores were distributed in different directions (Figure 3H, I). As a result, the patients in the low-risk group had better survival outcomes than those in the high-risk group (Figure 3J).

A

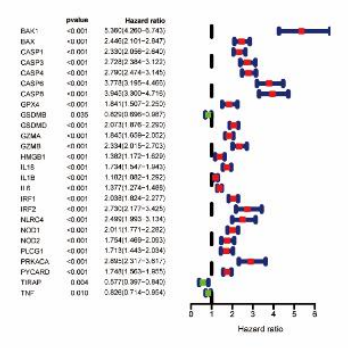

$\mathbf{E}$

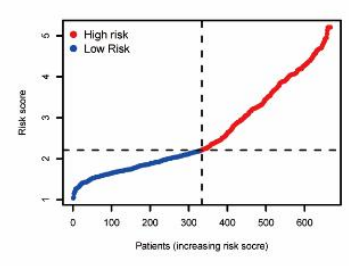

H

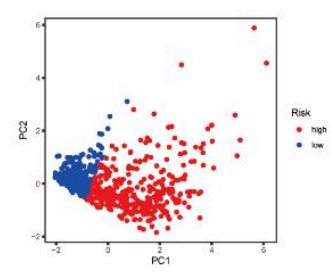

B

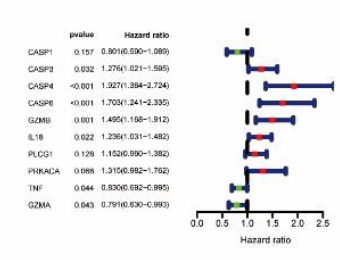

C

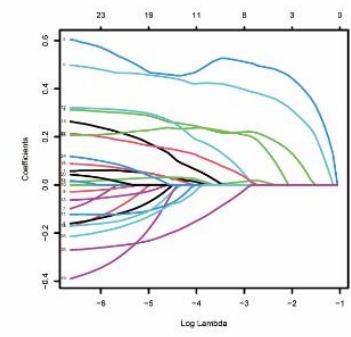

D

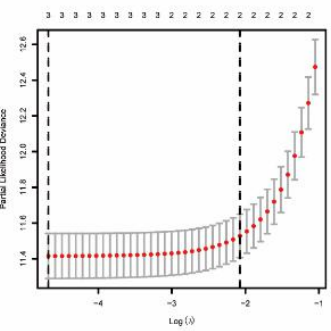

F

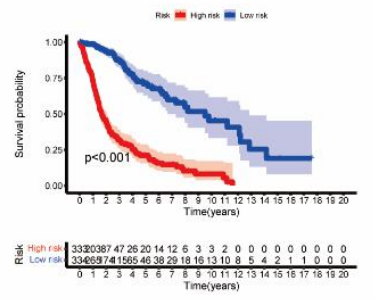

I

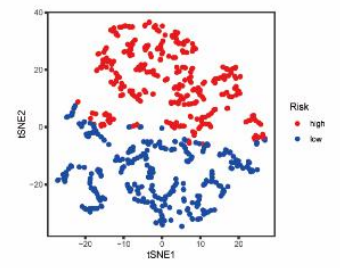

G

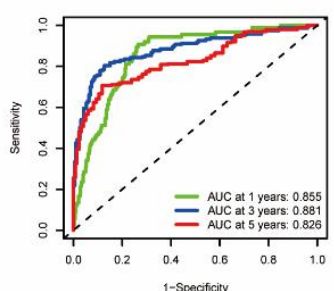

J

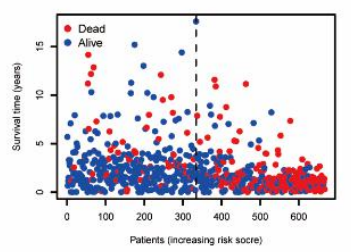


FIGURE 3 Prognostic analysis of the risk signature model. Forest plot of survival-related PRGs using univariate (A) and multivariable (B) Cox proportional hazards regression analysis. (C) Lasso regression of the 3 survival-related PRGs. (D) Plots of the 10-fold cross-validation in Lasso regression. (E) The risk score distribution of patients in the TCGA dataset. (F) Kaplan-Meier analysis for the OS of patients in the high- (red) and low-(blue) risk groups. (G) ROC curves for survival model predictive accuracy. (H) PCA plot of the TCGA cohort. (I) t-distributed Stochastic Neighbor Embedding (t-SNE) plot for patients. (J) Distribution of risk score, survival status, and survival time.

\section{Validation of the three-gene signature in the CGGA cohort}

A total of 693 glioma samples from the CGGA cohort (CGGA- mRNAseq693-clinical) was utilized as the test set in this study. Before proceeding further, we normalized RNA-sequencing expression data using the same formula. Based on the median cut-off value from the TCGA cohort, patients were divided into high-risk groups ( $\mathrm{n}=$ 409) and low-risk groups $(\mathrm{n}=248)$ (Figure 4A). Patients in the high-risk group had worse survival outcomes than those in the low-risk group (Figure 4B). The PCA and t-SNE exhibited satisfactory separation results between the two subgroups (Figure 4C, D). The overall survival of glioma patients in the low-risk group was significantly better than that in the control group (Figure 4E; $P<0.001$ ). In addition, the AUC values of the prognostic model were $0.687,0.728$, and 0.740 at 1-, 3-, and 5- years, respectively (Figure 4F). Analysis of the prognostic prediction through pyroptosis-based classification manifested that the risk-score still had comparatively high performance, manifesting that the risk-score could accurately predict the clinical outcome of glioma patients.

A

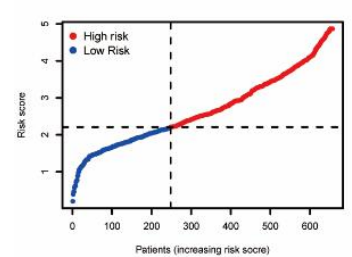

$\mathbf{E}$

\section{B}

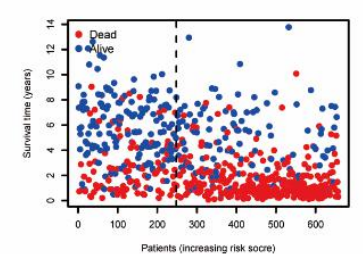

Pationts (increasing riak socce)

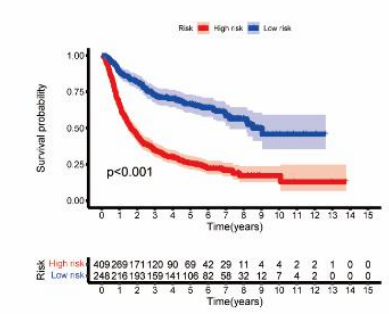

C

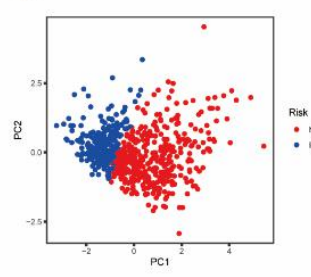

D

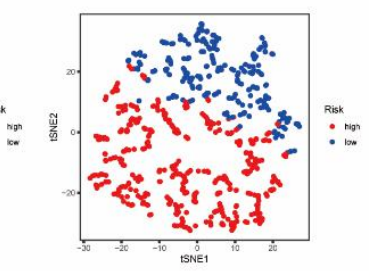

$\mathbf{F}$

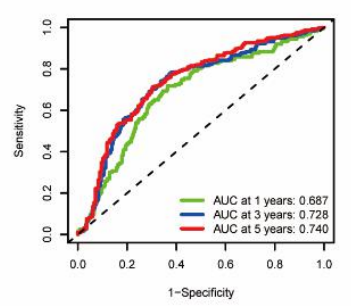

FIGURE 4 External validation of the risk signature model. (A) The risk score distribution of patients in the CGGA dataset. (B) Distribution of risk score, survival status, and survival time for each patient. (C) PCA plot of the CGGA cohort. (D) t-SNE plot for patients based on the risk score. (E) Kaplan-Meier survival analysis for 
the OS of glioma patients. (F) Time-dependent ROC curves.

\section{Independent prognostic value of the PRGs}

We then performed univariate and multivariable Cox proportional hazard regression to assess whether the pyroptosis risk model could be an independent prognostic factor for glioma patients. Univariate Cox regression analysis showed that age, grade, and risk score were correlated with prognosis. The higher the pyroptosis risk score, the worse the prognosis (HR: 2.793, 95\%CI: 2.477-3.148, $P<0.001$ Figure 5A; HR: 1.680, 95\% CI: $1.530-1.645, P<0.001$, Figure 5B). After adjusting for possible confounding factors, the multivariate analysis also revealed that the risk score was an independent prognostic factor (HR: 1.688, 95\% CI: 1.411-2.019, $P<0.001$ Figure 5C; HR: 1.325, 95\% CI: 1.194-1.471, $P<0.001$, Figure 5D) for patients with glioma. Moreover, we conducted a heatmap to evaluate the clinicopathological parameters of the TCGA cohort and observed statistically significant differences in patient age and tumor grade between the high-risk and low-risk subgroups. (Figure 5E, $P<0.001$ ).

Furthermore, we used immunohistochemistry to test CASP4, CASP6, and GZMB protein expression in glioma tissues and their counterparts, and the results were compared with the three DEG expression data from TCGA. We found that CASP4 proteins were highly expressed in glioma tissues than in normal brain tissues. Conversely, there was no CASP6 or GBMB staining in the glioma (Figure 5F, G, H).

A

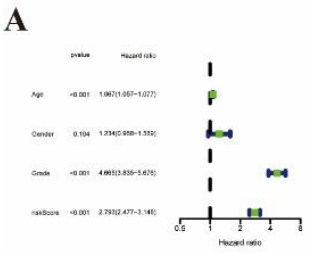

C

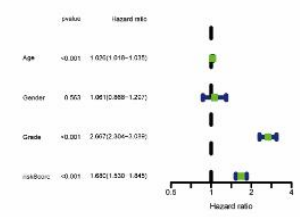

$\mathbf{E}$

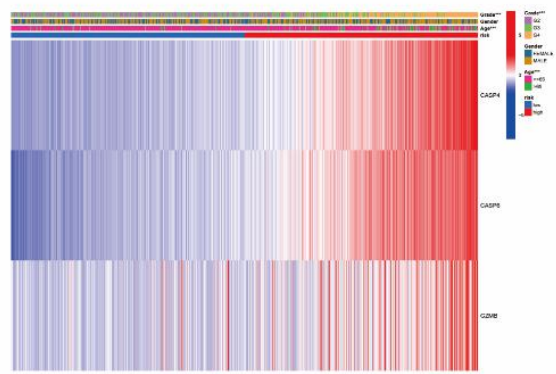

F

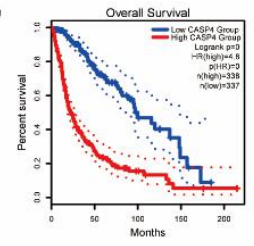

G

D
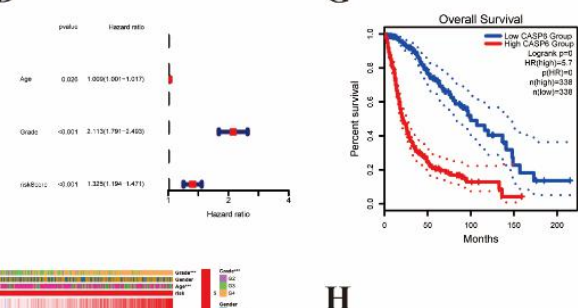

H

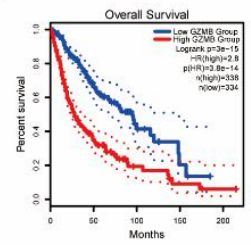

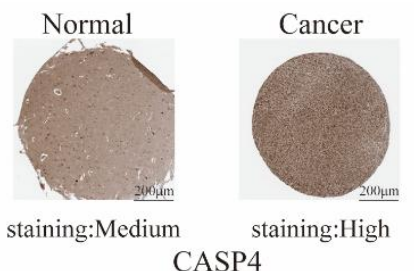

atibody:CAB03716

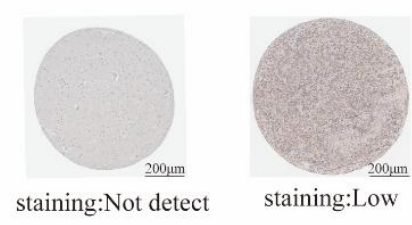

CASP6

Antibody:CAB003775

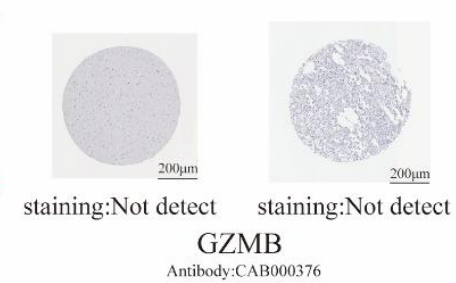

FIGURE 5 Independent prognostic analysis of clinicopathological features, risk scores, and immunohistochemical images in normal and tumor tissues.

(A, Green) Univariate and (B, Red) multivariate Cox regression analysis of the correlation between clinicopathological features and pyroptosis-based risk signature in the TCGA 
cohort. (C) Univariate and (D) multivariate Cox regression analysis in the CGGA cohort. (E) Heatmap (red: high expression; blue: low expression) for the correlation between the risk scores and clinicopathologic characteristics. Comparison of three genes expression between tumor and normal tissues (middle) and immunohistochemistry images in tumor and normal tissues (right). (F) CASP4. (G) CASP6. (H) GZMB.

\section{Functional enrichment and immune characteristics analysis}

To clarify the function of pyroptosis-related genes between the two subgroups classified derived from the risk model, we used the "limma" R package with $|\log 2 \mathrm{FC}|$ $\geq 1$ and FDR $<0.05$ to extract the DEGs in the TCGA cohort. In total, 1453 DEGs between the high- and low-risk groups were identified. And among them, 846 genes were upregulated, while the other 607 genes were downregulated (Table S7). According to these DEGs, GO functional annotation and KEGG pathway analyses were then performed. The DEGs were mainly enriched in immune processes such as neutrophil activation, neutrophil degranulation, neutrophil activation, and involved in immune response in GO analysis (Figure 6A). Additionally, KEGG pathway analyses showed that the DEGs were mainly involved in the Phagosome, Proteoglycans in cancer, ECM-receptor interaction, Focal adhesion, Cell adhesion molecules, and AGE-RAGE signaling pathway (Figure 6B).

To find out the relationship between immune cell infiltrations and risk scores in the prognostic risk model, the scores of 16 immune cell subsets and 13 immune-related pathways or functions were quantified between immune status and risk scores by employing the ssGSEA method in R package GSVA. Remarkably, the enrichment scores of distinct immune cell types (aDCs, B_cells, CD8+_T_cells, Macrophages, NK-cells, T-helper-cells, Th1-cells, TIL, and Treg) were considerably different between the two subgroups (Figure 6C, E; $p<0.05$ ). Moreover, all 13 immune-related pathways were also different between the low- and high-risk groups (Figure 6D, F; $P<0.001$ ). 
A

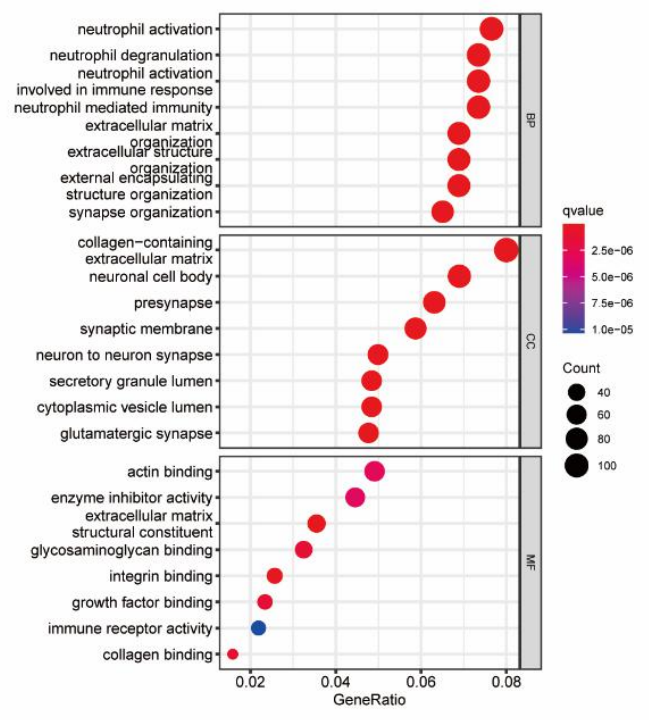

C

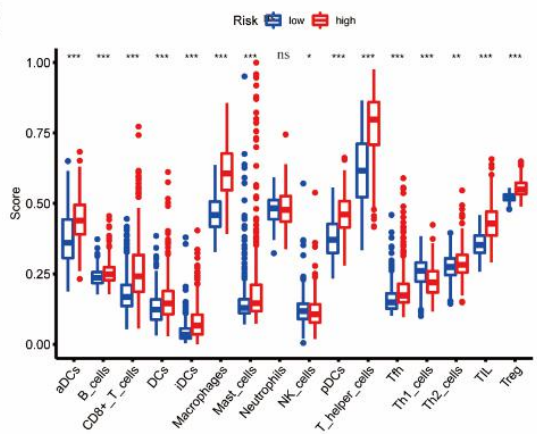

$\mathbf{E}$

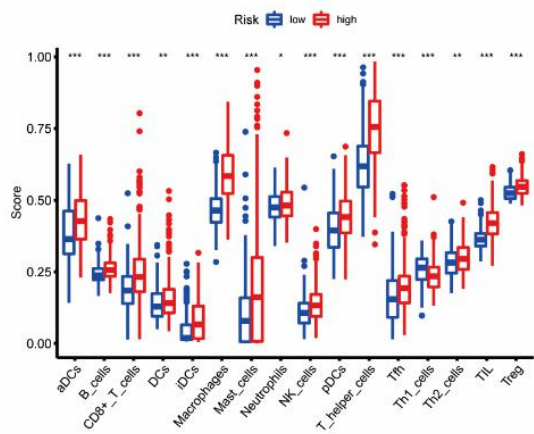

B

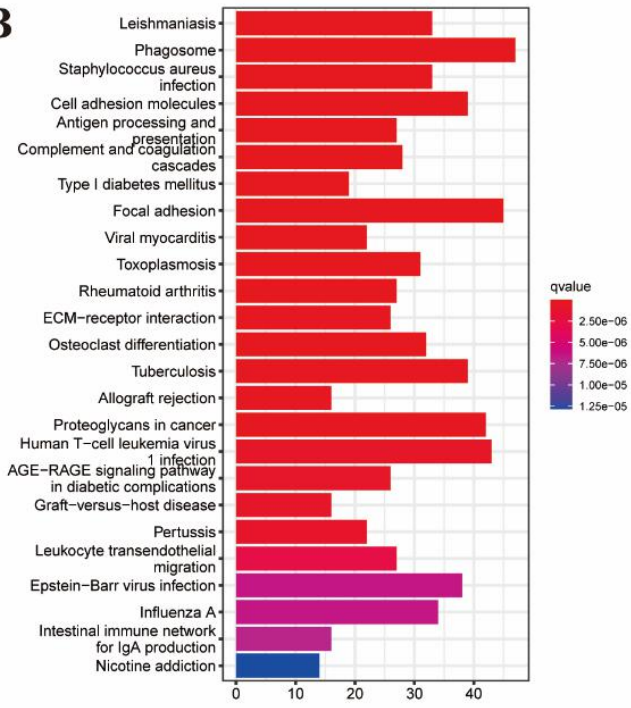

D

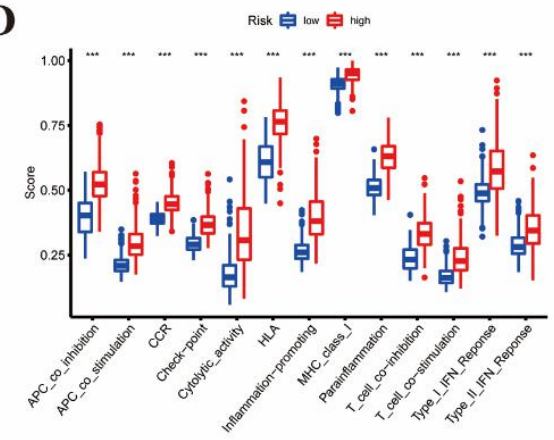

F

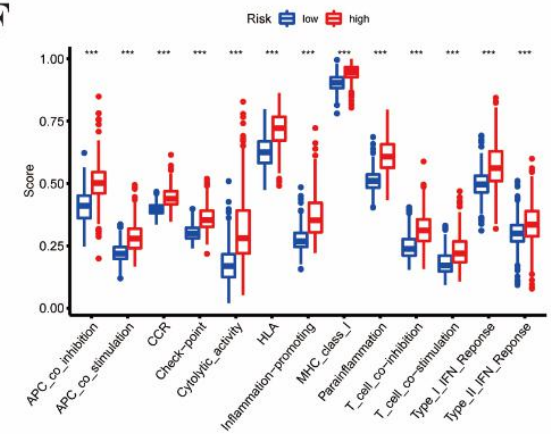

FIGURE 6 Functional enrichment analysis and Comparison of the immune response between two subgroups. (A) The enriched item in GO analysis. (B) The enriched item in KEGG analysis. The larger the bubble, the more genes enriched. Box plots of the ssGSEA scores of 16 immune cells (C) and 13 immune-related pathways (D) in the TCGA cohort (red, high-risk group; green, low-risk group). (E, F) Comparison of the enrichment scores between high-risk (red box) and low- (blue box) groups in the CGGA cohort.

\section{Comprehensive analysis of the risk-score in glioma}

Next, we examined whether risk-score had any guiding value for clinical prevention and treatment, especially promising immunotherapy. Tumor microenvironment analysis was performed, we found that the ImmuneScore and StromalScore from the 
ESTIMATE algorithm increased as the risk-score increased, while the TumorPurity exhibited the opposite trend (Figure 7A). These results revealed that the high-score group induced a stronger cellular immune response. The correlation between 6 types of immune checkpoint-related genes and risk-score was analyzed using the CIBERSORTx. Among them, CD274, PDCD1LG2, and HAVCR2 were significantly positively correlated with risk-score (Figure 7B). Figure 7C showed that the risk-score positively correlated with tumor-infiltrating immune cells (TIICs). We also investigated the relationship between the abundance of 22 immune infiltrating cells and the expression of three genes in the proposed model. We observed that $\mathrm{T}$ cells CD8, T cells CD4 memory activated, Monocytes, and Macrophages were significantly associated with three genes (Figure 7D). Additionally, we discovered that immune checkpoint-related genes were overexpressed in high-risk patients compared with low-risk patients, including PD-1, PD-L1, and CTLA-4 (Figure 7E).

Also, we synthesized the CSC index values and risk-score to evaluate the potential correlation between the risk-score and CSC in glioma. The linear correlation between the CSC index and risk-score was performed in Figure 7F-G. We discovered that risk-score was positively associated with the DNAss index $(\mathrm{R}=0.56, \mathrm{p}<0.001)$, while the RNAss index was negatively related to risk-score $(\mathrm{R}=-0.68, \mathrm{p}<0.001)$, suggesting that glioma cells with higher risk-score had more differentiation degree and more distinct stem cell properties (Figure 7F, G). Accumulative epidemiological evidence shows that patients with high TMB levels may benefit from prophylactic immunotherapy because of a higher proportion of tumor-specific neoantigens. However, integrated analysis of TMB did not yield any significant differences between the two risk groups (Figure 7H). We next selected a new chemotherapy drug applied for the treatment of glioma to assess the sensitivity of metformin in high- and low-risk patients. Then we found a lower IC50 score in the high-risk group. The result showed that three PRGs were involved in drug sensitivity (Figure 7I). IPS, as a new predictor of response to cancer immunotherapy, is widely-applied to evaluate the immune status of patients. Our analysis found that anti-PD-1 therapy was better than anti-CTLA-4 therapy in glioma patients, with higher IPS in the high-risk group (Figure 7J-M). 

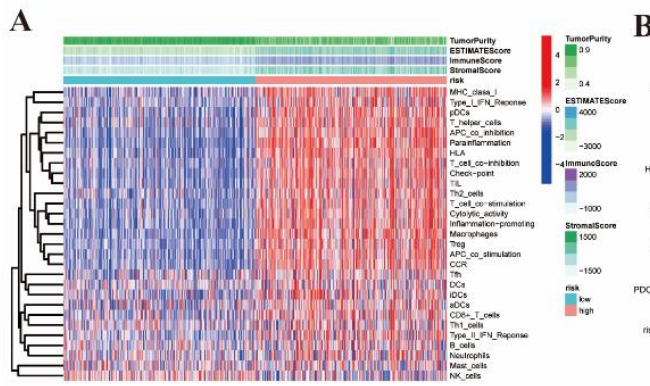

B
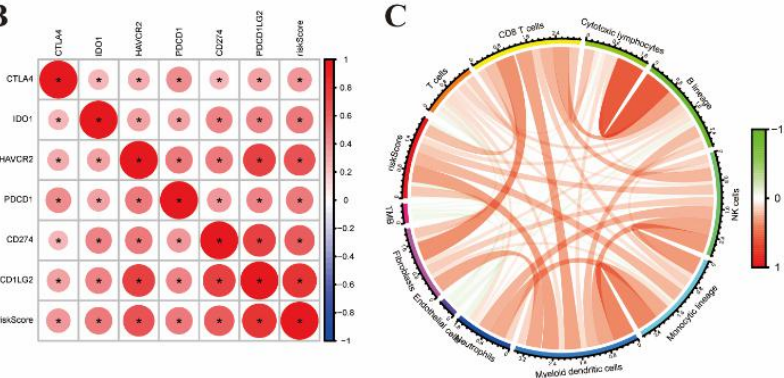

D
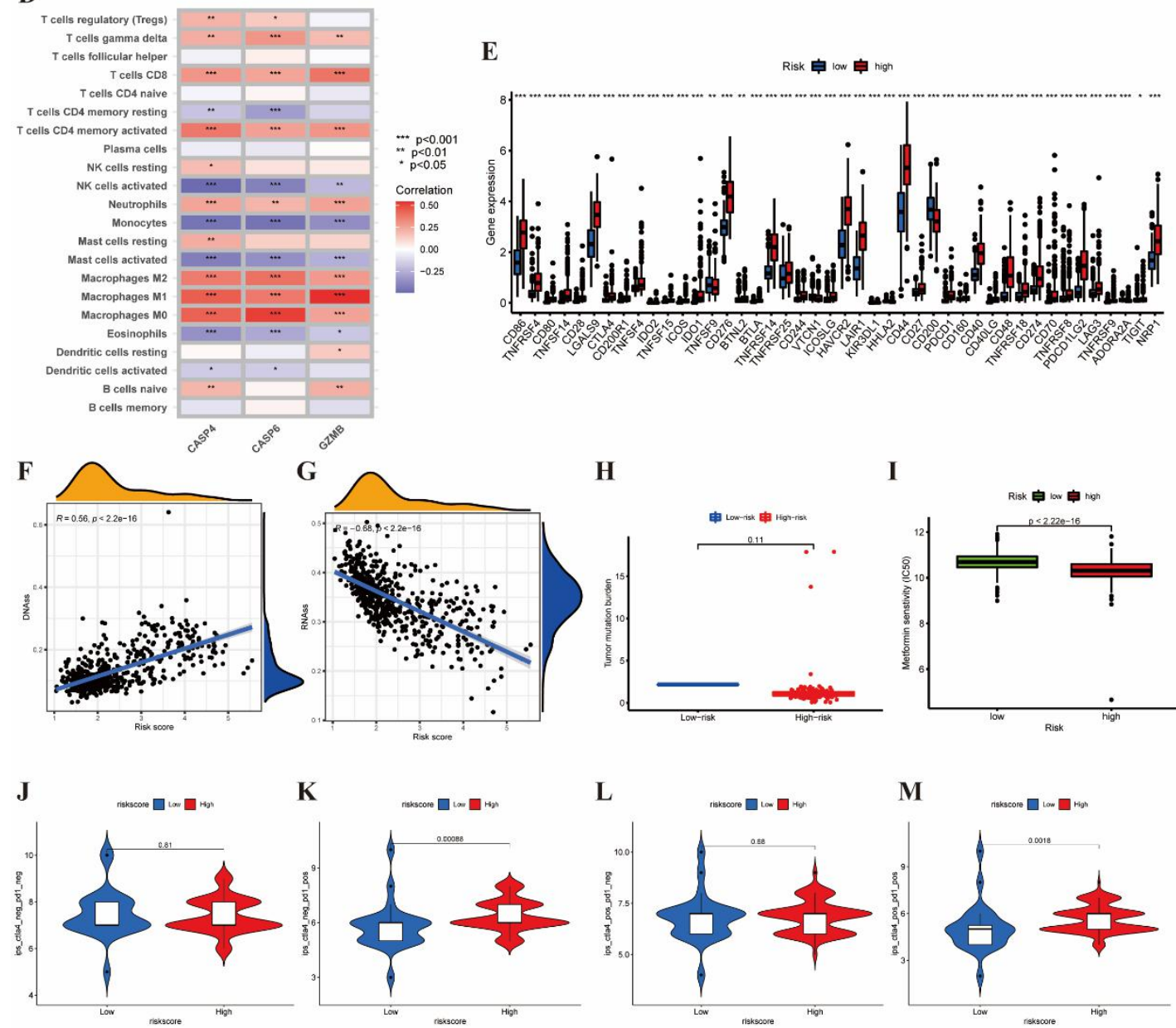

FIGURE 7 Relationship of risk-score with TME, CSC index, checkpoints, mutation, and drug susceptibility. (A) Heatmap of TME based on ESTIMATE algorithms between the two groups. (B) The correlation between 6 types of immune checkpoint-related genes and risk-score was analyzed. The size of the circle indicated a relevant correlation coefficient. (C) Correlations between TIIC levels, TMB, and risk-score. (D) Correlations between the abundance of 22 immune infiltrating cells and the expression of three genes. (E) Differential expression of immune checkpoint-related genes. $\mathrm{P}$ values were set as: $* \mathrm{P}<0.05 ; * * \mathrm{P}<0.01 ; * * * \mathrm{P}<0.001$. Relationships between risk score and cancer stem cell index. (F) DNAss. (G) RNAss. (H) Correlations between TMB and risk-score. (I) Relationships between chemotherapeutic sensitivity and risk-score. The difference of Immunophenoscore between two groups. (J) CTLA4 (-)/PD1 (-). (K) CTLA4 (-)/PD1 (+). (L) CTLA4 
$(+) /$ PD1 (-). (M) CTLA4 (+)/PD1 (+).

\section{Building a prognostic nomogram model for glioma}

Considering the inconvenience of risk-score in the clinical application of glioma patients, a nomogram clinical model was established to predict the survival probability (Figure 8A). The predictors included risk-score, PRS-type, Radio-status, Chemo-status, Grade, patient Age, and Gender. The predictive model indicated that the 3-year and 5-year OS rates could be predicted comparatively well in the CGGA cohort (Figure 8B, C). Next, clinical ROC and DCA analysis were performed to evaluate the effectiveness of the nomogram model, and the AUC of the Nomogram was 0.810 for 5 -year survival, indicating potential nomogram clinical model values of PRGs (Figure 8D, E).

A

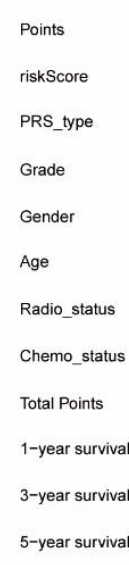

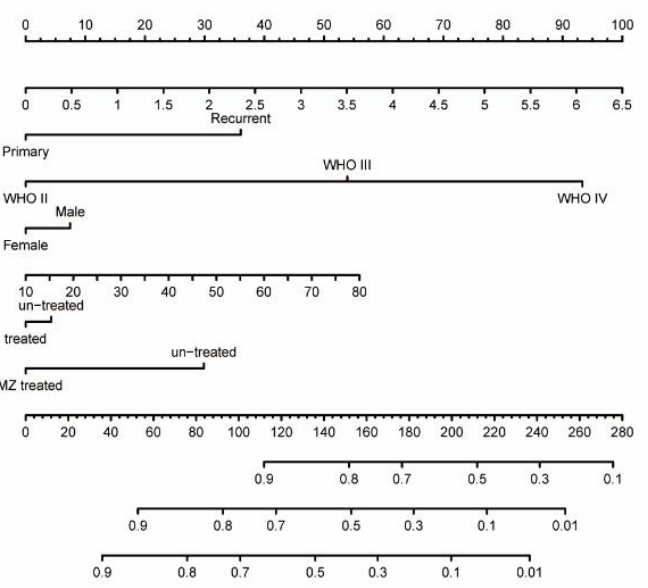

B

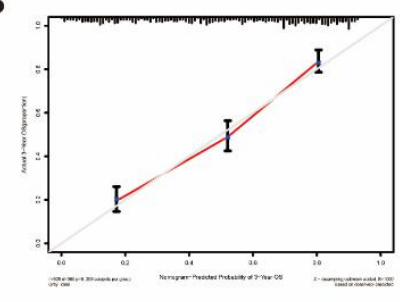

C

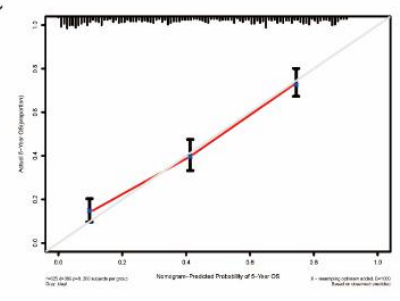

D

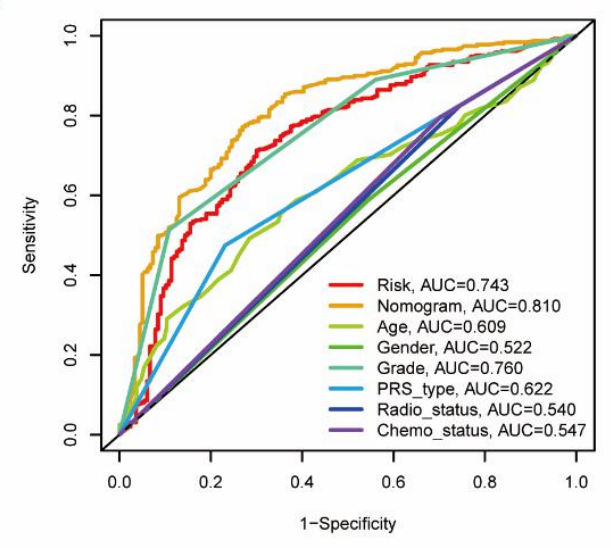

$\mathbf{E}$

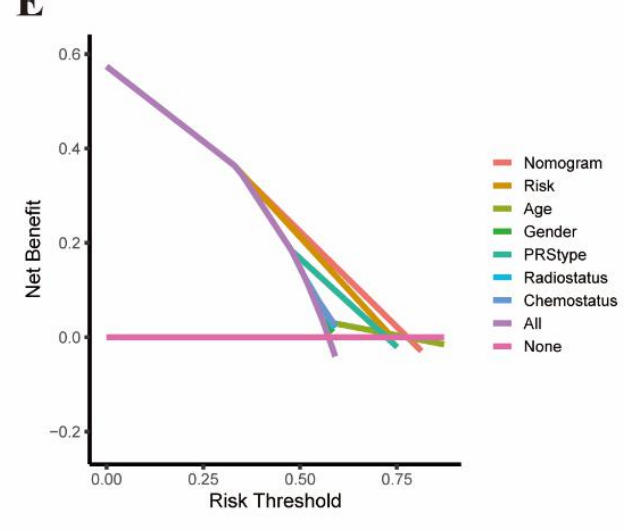

FIGURE 8 Establishment and application of the risk scoring model in CGGA cohort. (A) Prognostic nomogram for predicting the probability of 1-, 3-, and 5-years OS for patients with glioma in the CGGA cohort. (B, C) The calibration curves for the probabilities of 3- and 5- year OS. (D) The clinical ROC curves for the 5-year OS (E) Decision curve analysis for the 5-year OS. 


\section{DISCUSSION}

The results of this study revealed the overall alterations in PRGs at both genetic and transcriptional levels in glioma. Then two distinct molecular subtypes of glioma were identified based on 33 DEGs. Compared to subtype C1, glioma patients in subtype C2 had worse OS and PFS and more advanced clinicopathological features. There were also significant differences in TME characteristics between the two subtypes. Moreover, differences in mRNA transcriptome among different subtypes of pyroptosis were significantly correlated with PRGs. To further evaluate the prognostic characteristics of these PRGs, a 3 pyroptosis - related gene signature was constructed via Lasso Cox regression analysis and then verified that it performed well in the external dataset. We extracted the DEGs between the different risk groups and found that these DEGs were mainly associated with immune responses. It is also reasonable to speculate that pyroptosis may modulate the function of TME based on the results of functional enrichment analysis. In the present study, a lower risk-score was associated with immune activation, while a higher risk-score was associated with immune inhibition. We compared the activity of immune cell infiltration and immune-related pathways in the two groups and found that the immune cell infiltration levels and activation pathways of the low-risk group were generally lower than those of the high-risk group. Then we found that the characteristics of the abundance of the TIICs and the tumor microenvironment also differed significantly between the different risk groups. These findings indicate the key role of PRGs in the development and progression of glioma.

Recent studies have proved that B cells are also involved in immune activity (23, 24). Petitprez et al. reported that B cell enrichment was associated with resistance to anti-PD-1 checkpoint blockade in soft-tissue sarcomas and was an independent predictor for prolonged survival (25). Moreover, Helmink et al. showed that the expression of B cell-related genes MZB1 and IGLL5 in patients sensitive to ICB was significantly higher than those in non-responders (24). Meanwhile, the infiltration of B cells was correlated to a poor response to chemotherapeutics in patients with glioma (26). In medulloblastoma and pediatric glioblastoma, small molecule B-cell inhibitors can enhance tumor cell killing in combination with targeted chemotherapeutic agents (27). The results of these studies indicated that B cells may provide a new strategy for anti-tumor immunotherapy. In our present study, a significant difference was observed in B cells infiltration among the two molecular subtypes and different risk-score groups. The lower relative proportion of $\mathrm{B}$ cells in subtype $\mathrm{C} 1$ and low risk-score with the favorable OS. Consequently, tumor-infiltrating B cells promoted tumor progression in glioma, which is consistent with the results of previous studies. Tanaka et al. reported that Tregs infiltration can inhibit the anti-tumor immune response, which was correlated with an unsatisfied prognosis (28). This is consistent with our findings that compared with the low-risk group, patients with high risk-score have more Tregs in TME. These findings suggest that PRGs might be used as a predictor of immunotherapy response and clinical outcome in glioma. Thus, we 
established the effective and robust prognostic risk-score and regression analysis demonstrated its predictive power. The transcription levels of three PRGs included the risk-score in glioma samples were also explored. Patients with high- and low-risk groups showed significantly different clinical parameters, prognosis, immune checkpoints, TME, CSC index, and drug susceptibility. In the end, we constructed a quantitative nomogram model by integrating the clinicopathological characteristics and risk-score, which further facilitated the clinical application of the risk-score and improved the performance. This model can be used to stratify the prognosis of glioma patients, will help to better understand the molecular and functional mechanism of glioma, and will provide extensive new insights for targeted therapy.

So far, although it has been found that pyroptosis and apoptosis have certain similarities and crossover mechanisms, they have not been fully studied yet. Multiple modes of cell death are made up of numerous complex systems of order that both frequently coexist and interact with each other during tumor development (29). For example, CASP6 in our model is also considered to be a key regulator in apoptotic signaling pathways. Apoptosis usually features a complete plasma membrane with no contents release and no direct inflammatory response, whereas pyroptosis does the opposite characteristics (30). Apoptosis can be transformed into pyroptosis under the stimulation of pathogens. Pyroptosis plays several roles in tumors. It has the ability to reduce tumor growth and angiogenesis in liver cancer, skin carcinoma, and colorectal cancer $(31,32)$, but a double-way effect mechanism in breast cancer (33). Previous studies revealed that Caspase-6 (CASP6) is the executor of apoptosis. CASP6 is a critical regulator of innate immunity, host defense, and inflammasome activation. Besides, Zheng et al showed that the pyroptosis phenomenon of macrophages infected with seasonal influenza A virus (IAV) was reduced by conditionally knocking out CASP6, including the lysis of caspase- 1 and the reduction of IL-1 $\beta$ and IL-18 release (34). Caspase-4 (CASP4) participates in another pyroptosis pathway. GSDMD is specifically cleaved to ignite pyroptosis by CASP4 (35). Many studies have also confirmed the pyroptosis pathway induced by activated CASP4 in tumor cells (36). Zhang et al reported that GZMB can cleave gasdermin E and harness its pyroptosis to stimulate an effective immune response against immunologically cold tumors (37). With the deepening of molecular biology and tumor immunology research, immunotherapy offers a new targeted strategy to counteract tumors. This immunotherapy includes cell therapy, therapeutic antibodies, and immune checkpoint inhibitors (ICIs). Research on ICIs for PD-1, PD-L1, and CTLA-4 is booming, and clinical trials have demonstrated their efficacy and safety $(38,39)$. ICIs have recently been utilized in the treatment of glioma (40). In our study, we identified that PD-1 and PD-L1 were expressed at higher levels in the subtype C2 and low risk-score groups. We concluded that patients with high risk-score have higher expression rates of PD-1, PD-L1 and might be predisposed to respond to ICIs. However, we found that the expression of CTLA-4 was different between the high- and low-risk groups, but the efficacy between the two groups did not show any significant differences.

The comprehensive analysis of pyroptosis revealed a wide range of regulatory mechanisms, which affect the clinicopathological characteristics, prognosis, and 
tumor immune microenvironment. We also determined the therapeutic potential of PRGs in cancer-stem-cell-targeted immunotherapy. Our study highlights the clinical implications of pyroptosis and provides a novel insight for guiding precise immunotherapy strategies for glioma patients. Pyroptosis blockers are being developed, but there is not enough evidence to support it. Thus, our signature identifies potential new therapeutic targets for immunotherapy, which may be an effective complementary treatment direction to improve the clinical outcome and prognosis of patients with glioma.

Our study had some limitations. All data management and statistical analyses were performed solely from online databases, and all glioma samples used in this study were retrospectively collected. Thus, large-scale prospective cohort studies and further in-depth experimental studies are needed to extend and confirm these findings.

\section{ABBREVIATIONS}

GBM, Glioblastoma; LGG, low-grade glioma; TCGA, The Cancer Genome Atlas; TME, tumor microenvironment; TCGA, the cancer genome atlas; GO, gene ontology; KEGG, Kyoto Encyclopedia of Genes and Genomes; OS, overall survival; DFS, disease-free survival; TIICs, tumor-infiltrating immune cells; ROC, Receiver Operating Characteristic; AUC, Area Under the Curve.

\section{AUTHOR CONTRIBUTIONS}

$\mathrm{JH}$ and MZ designed the study and carried out experiments. JJ, JY, and JL analyzed the data. JH, JJ, and WZ wrote the draft of the manuscript. MZ edited the manuscript. All the authors read and approved the final version of the manuscript.

\section{CONFLICTS OF INTEREST}

All authors declared that there were no conflicts of interest with the contents of this article.

\section{ACKNOWLEDGMENTS}

The authors would like to thank all members of the Laboratory of Neurosurgery at the Second Xiangya Hospital for technical assistance.

\section{FUNDING}

This work was funded by the National Natural Science Foundation of China (Grant No.82001275) and the Natural Science Foundation of Hunan Province, China (Grant No. 2019JJ40417). 


\section{REFERENCE}

1. Chen W, Zheng R, Baade PD, Zhang S, Zeng H, Bray F, et al. Cancer statistics in China, 2015. CA Cancer J Clin. 2016;66(2):115-32.

2. Fitzmaurice C, Abate D, Abbasi N, Abbastabar H, Abd-Allah F, Abdel-Rahman O, et al. Global, Regional, and National Cancer Incidence, Mortality, Years of Life Lost, Years Lived With Disability, and Disability-Adjusted Life-Years for 29 Cancer Groups, 1990 to 2017: A Systematic Analysis for the Global Burden of Disease Study. JAMA Oncol. 2019;5(12):1749-68.

3. Deng L, Zheng W, Dong X, Liu J, Zhu C, Lu D, et al. Chemokine receptor CXCR7 is an independent prognostic biomarker in glioblastoma. Cancer Biomark. 2017;20(1):1-6.

4. Bush NA, Chang SM, Berger MS. Current and future strategies for the treatment of glioma. Neurosurg Rev. 2017;40(1):1-14.

5. Fang Y, Tian S, Pan Y, Li W, Wang Q, Tang Y, et al. Pyroptosis: A new frontier in cancer. Biomed Pharmacother. 2020;121:109595.

6. Broz P, Pelegrín P, Shao F. The gasdermins, a protein family executing cell death and inflammation. Nat Rev Immunol. 2020;20(3):143-57.

7. Yu P, Zhang X, Liu N, Tang L, Peng C, Chen X. Pyroptosis: mechanisms and diseases. Signal Transduct Target Ther. 2021;6(1):128.

8. Kolb R, Liu GH, Janowski AM, Sutterwala FS, Zhang W. Inflammasomes in cancer: a double-edged sword. Protein Cell. 2014;5(1):12-20.

9. Hou J, Zhao R, Xia W, Chang CW, You Y, Hsu JM, et al. PD-L1-mediated gasdermin C expression switches apoptosis to pyroptosis in cancer cells and facilitates tumour necrosis. Nat Cell Biol. 2020;22(10):1264-75.

10. Xi G, Gao J, Wan B, Zhan P, Xu W, Lv T, et al. GSDMD is required for effector CD8(+) T cell responses to lung cancer cells. Int Immunopharmacol. 2019;74:105713.

11. Zhou Z, He H, Wang K, Shi X, Wang Y, Su Y, et al. Granzyme A from cytotoxic lymphocytes cleaves GSDMB to trigger pyroptosis in target cells. Science. 2020;368(6494).

12. Yang L, Lin PC. Mechanisms that drive inflammatory tumor microenvironment, tumor heterogeneity, and metastatic progression. Semin Cancer Biol. 2017;47:185-95.

13. Orning P, Lien E, Fitzgerald KA. Gasdermins and their role in immunity and inflammation. J Exp Med. 2019;216(11):2453-65.

14. Erkes DA, Cai W, Sanchez IM, Purwin TJ, Rogers C, Field CO, et al. Mutant BRAF and MEK Inhibitors Regulate the Tumor Immune Microenvironment via Pyroptosis. Cancer Discov. 2020;10(2):254-69.

15. The Genotype-Tissue Expression (GTEx) project. Nat Genet. 2013;45(6):580-5.

16. Zhao Z, Zhang KN, Wang Q, Li G, Zeng F, Zhang Y, et al. Chinese Glioma Genome Atlas (CGGA): A Comprehensive Resource with Functional Genomic Data from Chinese Glioma Patients. Genomics Proteomics Bioinformatics. 2021;19(1):1-12.

17. Conesa A, Madrigal P, Tarazona S, Gomez-Cabrero D, Cervera A, McPherson A, et al. A survey of best practices for RNA-seq data analysis. Genome Biol. 2016;17:13.

18. Safran M, Dalah I, Alexander J, Rosen N, Iny Stein T, Shmoish M, et al. GeneCards Version 3: the human gene integrator. Database (Oxford). 2010;2010:baq020.

19. Xia X, Wang X, Cheng Z, Qin W, Lei L, Jiang J, et al. The role of pyroptosis in cancer: pro-cancer or pro-"host"? Cell Death Dis. 2019;10(9):650. 
20. Karki R, Kanneganti TD. Diverging inflammasome signals in tumorigenesis and potential targeting. Nat Rev Cancer. 2019;19(4):197-214.

21. Newman AM, Liu CL, Green MR, Gentles AJ, Feng W, Xu Y, et al. Robust enumeration of cell subsets from tissue expression profiles. Nat Methods. 2015;12(5):453-7.

22. Rooney MS, Shukla SA, Wu CJ, Getz G, Hacohen N. Molecular and genetic properties of tumors associated with local immune cytolytic activity. Cell. 2015;160(1-2):48-61.

23. Cabrita R, Lauss M, Sanna A, Donia M, Skaarup Larsen M, Mitra S, et al. Tertiary lymphoid structures improve immunotherapy and survival in melanoma. Nature. 2020;577(7791):561-5.

24. Helmink BA, Reddy SM, Gao J, Zhang S, Basar R, Thakur R, et al. B cells and tertiary lymphoid structures promote immunotherapy response. Nature. 2020;577(7791):549-55.

25. Petitprez F, de Reyniès A, Keung EZ, Chen TW, Sun CM, Calderaro J, et al. B cells are associated with survival and immunotherapy response in sarcoma. Nature. 2020;577(7791):556-60.

26. Wu L, Bernal GM, Cahill KE, Pytel P, Fitzpatrick CA, Mashek H, et al. BCL3 expression promotes resistance to alkylating chemotherapy in gliomas. Sci Transl Med. 2018;10(448).

27. Levesley J, Steele L, Brüning-Richardson A, Davison A, Zhou J, Ding C, et al. Selective BCL-XL inhibition promotes apoptosis in combination with MLN8237 in medulloblastoma and pediatric glioblastoma cells. Neuro Oncol. 2018;20(2):203-14.

28. Tanaka A, Sakaguchi S. Regulatory $\mathrm{T}$ cells in cancer immunotherapy. Cell Res. 2017;27(1):109-18.

29. Fritsch M, Günther SD, Schwarzer R, Albert MC, Schorn F, Werthenbach JP, et al. Caspase-8 is the molecular switch for apoptosis, necroptosis and pyroptosis. Nature. 2019;575(7784):683-7.

30. Khan I, Yousif A, Chesnokov M, Hong L, Chefetz I. A decade of cell death studies: Breathing new life into necroptosis. Pharmacol Ther. 2021;220:107717.

31. Zaki MH, Vogel P, Body-Malapel M, Lamkanfi M, Kanneganti TD. IL-18 production downstream of the Nlrp3 inflammasome confers protection against colorectal tumor formation. J Immunol. 2010;185(8):4912-20.

32. Ellis LZ, Liu W, Luo Y, Okamoto M, Qu D, Dunn JH, et al. Green tea polyphenol epigallocatechin-3-gallate suppresses melanoma growth by inhibiting inflammasome and IL-1 $\beta$ secretion. Biochem Biophys Res Commun. 2011;414(3):551-6.

33. Chen LC, Wang LJ, Tsang NM, Ojcius DM, Chen CC, Ouyang CN, et al. Tumour inflammasome-derived IL-1 $\beta$ recruits neutrophils and improves local recurrence-free survival in EBV-induced nasopharyngeal carcinoma. EMBO Mol Med. 2012;4(12):1276-93.

34. Zheng M, Karki R, Vogel P, Kanneganti TD. Caspase-6 Is a Key Regulator of Innate Immunity, Inflammasome Activation, and Host Defense. Cell. 2020;181(3):674-87.e13.

35. Shi J, Zhao Y, Wang K, Shi X, Wang Y, Huang H, et al. Cleavage of GSDMD by inflammatory caspases determines pyroptotic cell death. Nature. 2015;526(7575):660-5.

36. Qiao L, Wu X, Zhang J, Liu L, Sui X, Zhang R, et al. $\alpha$-NETA induces pyroptosis of epithelial ovarian cancer cells through the GSDMD/caspase-4 pathway. Faseb j. 2019;33(11):12760-7.

37. Zhang Z, Zhang Y, Lieberman J. Lighting a Fire: Can We Harness Pyroptosis to Ignite Antitumor Immunity? Cancer Immunol Res. 2021;9(1):2-7.

38. Yaghoubi N, Soltani A, Ghazvini K, Hassanian SM, Hashemy SI. PD-1/ PD-L1 blockade as a novel treatment for colorectal cancer. Biomed Pharmacother. 2019;110:312-8.

39. Rotte A. Combination of CTLA-4 and PD-1 blockers for treatment of cancer. J Exp Clin Cancer Res. 2019;38(1):255. 
40. Romani M, Pistillo MP, Carosio R, Morabito A, Banelli B. Immune Checkpoints and Innovative Therapies in Glioblastoma. Front Oncol. 2018;8:464. 


\section{Supplementary Files}

This is a list of supplementary files associated with this preprint. Click to download.

- SupplementaryFigures.docx

- SupplementaryTables.xlsx 